\title{
Fatou components whose boundaries have a common curve
}

\author{
by \\ Shunsuke Morosawa (Kochi)
}

\begin{abstract}
We show that the Fatou components of a certain transcendental entire function have a common curve in their boundaries.
\end{abstract}

1. Introduction. The Julia sets of holomorphic dynamics are always complicated except a few holomorphic maps, for example, $z \mapsto z^{2}$. The idea of hyperbolicity is one of the most important concepts in studying holomorphic dynamics. A rational function is called hyperbolic if the orbit of every critical point converges to an attracting periodic orbit; equivalently, the function is expanding with respect to the Euclidean metric on a neighborhood of the Julia set. This yields nice properties on Julia sets. For example, if the Julia set of a hyperbolic rational function is connected, then it is locally connected. Furthermore, the argument in the proof of $[6$, Theorem 3] shows that, for a hyperbolic rational function with the connected Julia set, if the boundaries of two Fatou components have a common curve, then the Fatou set consists of two components and the Julia set is a Jordan curve. Consequently, the Fatou set of the two-fold iterate consists of two completely invariant components. We easily define the hyperbolicity for a transcendental entire function: the orbit of every singular point converges to an attracting periodic orbit. In [10], hyperbolic meromorphic functions were investigated and it was shown that they are expanding. Nevertheless, hyperbolic transcendental entire functions are not uniformly expanding because the Julia sets of transcendental entire functions are always unbounded.

In this note, we show that the Fatou components of a certain hyperbolic complex error function have a common curve in their boundaries. The precise statement and its proof are given in Section 3. Complex error functions are defined in Section 2. The fundamental properties of dynamics of transcendental entire functions we use in this note can be found in $[8]$.

2000 Mathematics Subject Classification: Primary 37F10; Secondary 30D05. 
Acknowledgements. The author is grateful to the referee for valuable suggestions. He thanks the Japan Society for the Promotion of Science, Grant No. 14540179, 2003 for support.

2. Complex error functions. A complex error function is a transcendental entire function given by

$$
f_{a, b}(z)=a \int_{0}^{z} e^{-w^{2}} d w+b
$$

with $a \in \mathbb{C} \backslash\{0\}$ and $b \in \mathbb{C}$. It has two asymptotic values $\pm a \sqrt{\pi} / 2+b$ and no other singular values. A transcendental entire function with finitely many singular values has neither a Baker domain nor a wandering domain (see [1], [3] and [4]). Hence the Fatou set of a complex error function contains neither a Baker domain nor a wandering domain. This implies that points tending to infinity under the iteration belong to the Julia set. Furthermore, a complex error function has at most two non-repelling cycles, since every non-repelling cycle has a relationship with singular values (see [3] and [8]). We also remark that a transcendental entire function has at most one completely invariant component (see [3] and [8]).

A complex error function is unique up to the involution which carries it into the map

$$
-f_{a, b}(-z)=a \int_{0}^{z} e^{-w^{2}} d w-b=f_{a,-b}(z) .
$$

Thus the two numbers $a$ and $B=b^{2}$ form a complete set of coordinates for the moduli space. A complex error function given by

$$
f_{a, \sqrt{B}}(z)=a \int_{0}^{z} e^{-w^{2}} d w+\sqrt{B}
$$

with $a \in \mathbb{R} \backslash\{0\}$ and $B \in \mathbb{R}$, is called a real error function in this note. We put $b=\sqrt{|B|}$. If $B>0$, then

$$
f_{a, \sqrt{B}}(z)=a \int_{0}^{z} e^{-w^{2}} d w+b
$$

and if $B \leq 0$, then

$$
f_{a, \sqrt{B}}(z)=a \int_{0}^{z} e^{-w^{2}} d w+i b .
$$

The action of every real error function with $B>0$ is symmetric with respect to the real axis, and for $B \leq 0$ it is symmetric with respect to the imaginary axis. 
Assume $B>0$. If $a>0$, then $f_{a, \sqrt{B}}$ is increasing on the real axis, convex on the negative halfaxis and concave on the positive halfaxis, since $f_{a, \sqrt{B}}^{\prime}(x)=a e^{-x^{2}}$ and $f_{a, \sqrt{B}}^{\prime \prime}(x)=-2 a x e^{-x^{2}}$. Furthermore, its values lie between $a \sqrt{\pi} / 2+\sqrt{B}$ and $-a \sqrt{\pi} / 2+\sqrt{B}$. Hence its Fatou set consists of one of the following: a completely invariant component with an attracting fixed point, two basins of attracting fixed points or a basin of an attracting fixed point and a basin of a parabolic fixed point. Moreover, $f_{a, \sqrt{B}}$ has at most three fixed points on the real axis. If it has only one fixed point, then it is an attracting fixed point and the orbit of each asymptotic value converges to it. If $f_{a, \sqrt{B}}$ has three fixed points, then one is repelling and the others are attracting. If the graph is tangent to the diagonal, then the tangent point is on the negative halfaxis since $\sqrt{B}>0$. Hence it is a parabolic fixed point with multiplicity two. In this case, $f_{a, \sqrt{B}}$ has also an attracting fixed point on the positive halfaxis.

If $a<0$, then the function is decreasing. Furthermore, it is concave on the negative halfaxis and convex on the positive halfaxis. Its values lie in the same interval as in the case $a>0$. Hence its Fatou set consists of one of the following: a completely invariant component with an attracting fixed point, a basin of an attracting cyclic point with period two or a basin of a rationally indifferent fixed point with multiplier -1 . Moreover, $f_{a, \sqrt{B}}$ has only one fixed point with negative multiplier on the real axis. If its multiplier is in $(-1,0)$, it is an attracting fixed point and the orbit of each asymptotic value converges to it. If its multiplier is -1 , then it is an inflection point of $f_{a, \sqrt{B}}^{2}$. This implies that it is a parabolic fixed point of $f_{a, \sqrt{B}}^{2}$ with multiplicity 3 . If its multiplier is in $(-\infty,-1)$, then $f_{a, \sqrt{B}}^{2}$ has one repelling fixed point and two attracting fixed points.

In any case above, the real axis is contained in the Fatou set except at most one point, which is a repelling fixed point or a rationally indifferent fixed point. Hence both asymptotic values and asymptotic paths for both asymptotic values are contained in the immediate basins.

For $B \leq 0$, the parameter space $(a, B)$ is much more complicated and is investigated in [7] and [9].

It is well known that every immediate basin of an attracting cycle contains at least one singular value. The proof of this claim (for example, see [8, Theorem 2.4.1]) shows that if an immediate basin contains only asymptotic values as singular values, then it also contains at least one asymptotic path for one of the asymptotic values in it.

Now we consider real error functions with $B \leq 0$. The symmetry of the action with respect to the imaginary axis determines the Fatou set in the following sense. If $f_{a, i \sqrt{|B|}}$ has only one attracting fixed point, then the attract- 
ing fixed point exists on the imaginary axis. The immediate basin contains both asymptotic values and hence both asymptotic paths. Consequently, it is a completely invariant component. If $f_{a, i \sqrt{|B|}}$ has two attracting fixed points, then one is in the left halfplane and the other in the right halfplane. Each immediate basin contains an asymptotic value and its asymptotic path. Assume $f_{a, i \sqrt{|B|}}$ has only one attracting cycle with period two. If a component of the immediate basin contains only one asymptotic value, then the other component also contains the other asymptotic value. It follows that one component of the immediate basin is contained in the right halfplane and the other in the left halfplane. Accordingly, each component contains an asymptotic path. If a component of the immediate basin contains both asymptotic values, then the other component contains asymptotic paths for both asymptotic values. The periodic points of the attracting cycle exist on the imaginary axis.

REMARK 1. Taniguchi defined a structurally finite transcendental entire function to be of the form

$$
z \mapsto \int_{a}^{z} P(w) e^{Q(w)} d w
$$

where $P$ and $Q$ are polynomials and $a$ is a constant. Hence every complex error function is structurally finite. In [12], he studied the topological structure of families of structurally finite transcendental entire functions.

\section{Theorem and proof}

THEOREM. Assume a real error function $f_{a, \sqrt{B}}$ has either two attracting fixed points or only one attracting cycle with period two. Then the boundaries of the components of the immediate basins contain a common curve.

Proof. The number of components stated in the Theorem is two. We abbreviate $f_{a, \sqrt{B}}$ to $f$. The asymptotic value whose real part is greater than that of the other is denoted by $a^{+}$, and the other by $a^{-}$.

CAse 1: $B \leq 0$. From the symmetry of the action of $f$, we see that the imaginary axis is forward invariant. We denote the imaginary axis by $I_{0}$. Take a disc $D$ centered at the origin containing both asymptotic values. Then $f^{-1}(\mathbb{C} \backslash D)$ consists of two components. One of them, say $E_{U}$, intersects $\{z \mid \Im z>y\}$ for arbitrarily large $y$, and the other, denoted by $E_{L}$, intersects $\{z \mid \Im z<y\}$ for arbitrarily small $y$. We also denote by $C_{U}$ and $C_{L}$ the boundaries of $E_{U}$ and $E_{L}$, respectively. Let

$$
g(z)=f_{1,0}(z)=\int_{0}^{z} e^{-w^{2}} d w, \quad K=g^{-1}\left(I_{0}\right) .
$$


We note that $f^{-1}\left(I_{0}\right)=K$ for an arbitrary real error function with $B \leq 0$. The imaginary axis, which is contained in $K$, intersects $C_{U}$ in a unique point, say $P_{U, 0}$, and intersects $C_{L}$ in a unique point, say $P_{L, 0}$. Every component of $K$ except $I_{0}$ intersects either $C_{U}$ or $C_{L}$ in two points. The points of $C_{U} \cap K$ in the right halfplane are labeled $P_{U, 1}, P_{U, 2}, \ldots$ in the order of increasing length along $C_{U}$, measured from the imaginary axis, and those in the left halfplane are labeled $P_{U,-1}, P_{U,-2}, \ldots$ in the same order. In a similar fashion we also label $P_{L, i}$ for $i \in \mathbb{Z} \backslash\{0\}$ the points of $C_{L} \cap K$. Hereafter, * means $U$ or $L$. The curve in $K$ which contains $P_{*, 2 i}$ for $i \in \mathbb{Z} \backslash\{0\}$ is denoted by $I_{*, i}$. We denote by $D_{*, i}$ for $i \in \mathbb{Z} \backslash\{0\}$ the complementary component of $I_{*, i}$ which does not intersect $K$.

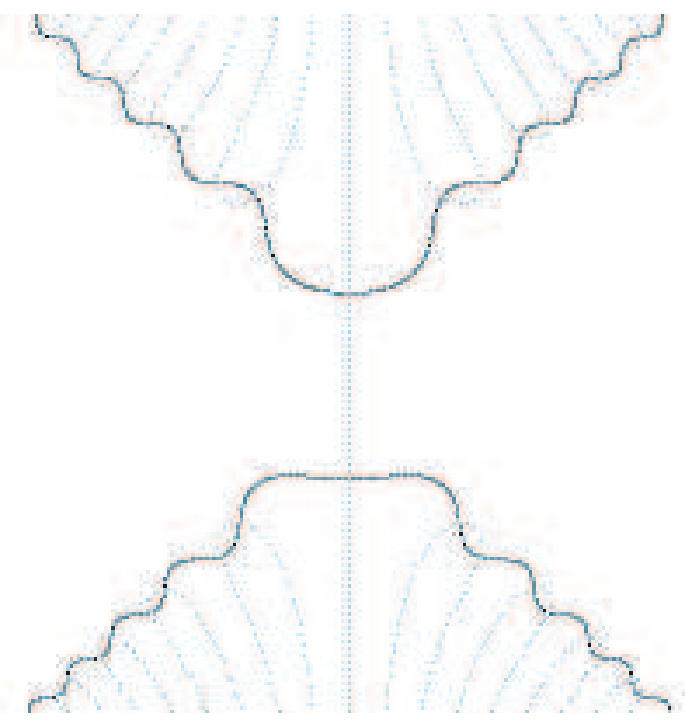

Fig. 1. The inverse image of $\partial D$ is black and the inverse image of the imaginary axis is gray.

First, we consider the case where $f$ has two attracting fixed points. It follows that $a>0$. Let $A^{+}$and $A^{-}$be the immediate basins containing $a^{+}$ and $a^{-}$, respectively. The symmetry of the Fatou set implies that the Julia set contains the imaginary axis. We show that $\partial A^{+}$and $\partial A^{-}$contain the imaginary axis. Each $D_{*, i}$ for $-i \in \mathbb{N}$ contains only one point of $f^{-1}\left(a^{+}\right)$. Furthermore, there exist no other points of $f^{-1}\left(a^{+}\right)$in $\mathbb{C} \backslash \bigcup_{i=-1}^{-\infty}\left(D_{U, i} \cup D_{L, i}\right)$. Therefore, a component of $f^{-1}\left(A^{+}\right)$other than $A^{+}$is contained in some $D_{*, i}$ for $-i \in \mathbb{N}$ and for $*=U$ or $L$. Similar results hold for $a^{-}$and $A^{-}$. Take an asymptotic path $\Gamma$ for $a^{+}$in $A^{+}$whose finite terminal point is $a^{+}$and which intersects $\partial D$ in only one point. We can choose a component of $f^{-1}(\Gamma)$ which is an asymptotic path for $a^{+}$. Such components are in $A^{+}$. Since both ends of the curve tend to infinity, we can denote it by $\gamma(t)$ for $t \in \mathbb{R}$, with 
$\lim _{|t| \rightarrow \infty}|\gamma(t)|=\infty$. We may assume $\gamma(t)$ for $t \in[0, \infty)$ is an asymptotic path for $a^{+}$. This implies $\lim _{t \rightarrow \infty} \Re(\gamma(t))=\infty$. Let $I_{*, i}^{-}$and $I_{*, i}^{+}$for $i \in \mathbb{Z} \backslash\{0\}$ be the subcurves of $I_{*, i}$ in $E_{*}$ whose finite terminal points are $P_{*, 2 i-1}$ and $P_{*, 2 i}$, respectively. We also denote $I_{0} \cap E_{U}$ and $I_{0} \cap E_{L}$ by $I_{U, 0}^{+}$and $I_{L, 0}^{+}$, respectively. Let $B_{*, i}$ be the domain bounded by $I_{*, i}^{-}, I_{*, i-1}^{+}$and $C_{*}$ for $i \in \mathbb{N}$. By the definition, $f\left(B_{*, i}\right)=(\bar{D})^{\mathrm{c}} \cap\{z \mid \Re z>0\}$. Conversely, every component of $f^{-1}\left((\bar{D})^{\mathrm{c}} \cap\{z \mid \Re z>0\}\right)$ in the right halfplane is equal to $B_{*, i}$ for some $i \in \mathbb{N}$ and for $*=U$ or $L$. Then we can choose two asymptotic paths $\gamma_{U}^{+}$and $\gamma_{L}^{+}$for $a^{+}$in $f^{-1}(\Gamma)$ satisfying $\gamma_{U}^{+} \cap B_{U, 1} \neq \emptyset$ and $\gamma_{L}^{+} \cap B_{L, 1} \neq \emptyset$. Consequently, one of the complementary components of $\gamma_{*}^{+}$contains $D_{*, i}$ for all $i \in \mathbb{N}$. By the argument similar to the above, we obtain the curve $\gamma_{*}^{-}$in $A^{-}$one of whose complementary components contains $D_{*, i}$ for all $-i \in \mathbb{N}$.

Take a point $w$ on $I_{0}$. If $w$ is not in $\partial A^{+}$, then it is not in $\partial A^{-}$from the symmetry of the Fatou set. Let $V$ be a small open disk centered at $w$ such that $V \cap A^{+}=\emptyset$ and $V \cap A^{-}=\emptyset$. Since $I_{0}$ is forward invariant, we have $f^{n}(V) \cap I_{0} \neq \emptyset$ for all $n \in \mathbb{N}$. A fundamental property of Julia sets shows that there exists $N$ such that $f^{N}(V) \cap A^{+} \neq \emptyset$ and $f^{n}(V) \cap A^{+}=\emptyset$ for all $n<N$. The symmetry of the action implies that also $f^{N}(V) \cap A^{-} \neq \emptyset$ and $f^{n}(V) \cap A^{-}=\emptyset$ for all $n<N$. Since $f^{N-1}(V) \cap A^{+}=\emptyset, f^{N-1}(V)$ must intersect a component of $f^{-1}\left(A^{+}\right)$other than $A^{+}$, which is contained in $D_{*, i}$ for some $-i \in \mathbb{N}$ and for $*=U$ or $L$. Because of $f^{N-1}(V) \cap I_{0} \neq \emptyset$, $f^{N-1}(V)$ intersects $\gamma_{*}^{-}$for $*=U$ or $L$, which yields $f^{N-1}(V) \cap A^{-} \neq \emptyset$. This is a contradiction. Hence $w$ is a boundary point of $A^{+}$and of $A^{-}$.
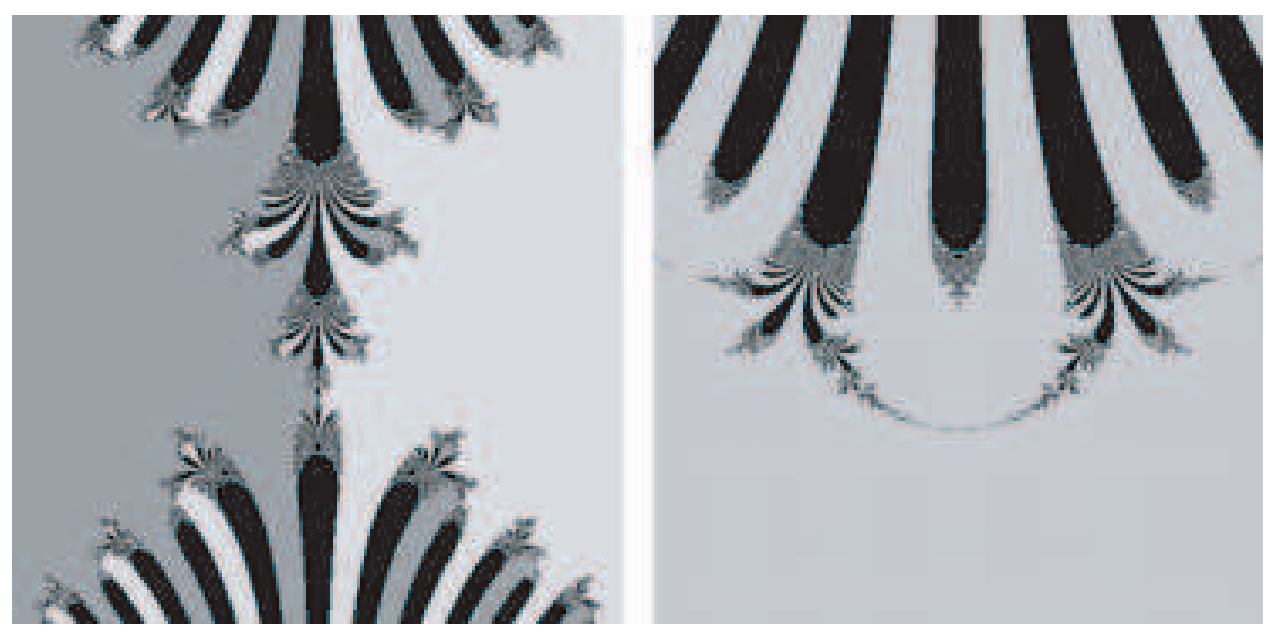

Fig. 2. The Julia sets of $f_{a, \sqrt{B}}$. Left: $f_{a, \sqrt{B}}$ with $(a, B)=(1.6,1.96)$ has two attracting fixed points. Right: $f_{a, \sqrt{B}}$ with $(a, B)=(-0.14,-3.61)$ has an attracting cycle with period two whose cyclic points are on the imaginary axis. 
Second, we consider the case where $f$ has an attracting cycle with period two, each component of whose immediate basin contains an asymptotic value. It follows that $a<0$. The Julia set again contains the imaginary axis. By an argument similar to the above, every component of $f^{-1}\left(A^{+}\right)$and of $f^{-1}\left(A^{-}\right)$except $A^{+}$and $A^{-}$is contained in $D_{*, i}$ for some $i \in \mathbb{Z} \backslash\{0\}$ and for $*=U$ or $L$. Furthermore, we can obtain the curves $\gamma_{*}^{ \pm}$which have the properties as above. Thus, we arrive at $\partial A^{+} \cap \partial A^{-} \supset I_{0}$ in the same way.

Finally, we consider the case where $f$ has an attracting cycle with period two, a component of whose immediate basin contains both asymptotic values. Let $A^{+}$be the component of the immediate basin containing both asymptotic values, and $A^{-}$the other component. By symmetry, $A^{+} \cap I_{0} \neq \emptyset$ and thus $A^{-} \cap I_{0} \neq \emptyset$. This implies $a<0$. We consider the case $b=\sqrt{|B|}>0$. Then $A^{+}$lies in the upper halfplane. Let $\gamma$ be a curve in $A^{+}$whose terminal points are the asymptotic values. We also assume $\gamma$ intersects $I_{0}$ in only one point. Since $a<0, f^{-1}\left(a^{+}\right)$is contained in the right halfplane and $f^{-1}\left(a^{-}\right)$ in the left halfplane. As before, each of $f^{-1}\left(a^{+}\right) \cup f^{-1}\left(a^{-}\right)$is contained in $D_{*, i}$ for $i \in \mathbb{Z} \backslash\{0\}$ and for $*=U$ or $L$, whose boundary is mapped onto the imaginary axis. Only one component of $f^{-1}(\gamma)$ intersects the imaginary axis, and tends to infinity on both sides. Other components have finite end points. If a finite end point of a component is in $f^{-1}\left(a^{+}\right)$, then the component is an asymptotic path for $a^{-}$. If a finite end point of a component is in $f^{-1}\left(a^{-}\right)$, then the component is an asymptotic path for $a^{+}$. Hence every point of $f^{-1}\left(a^{+}\right)$and of $f^{-1}\left(a^{-}\right)$is contained in $A^{-}$, which implies that $f^{-1}\left(A^{+}\right)=A^{-}$. Since the imaginary axis is not contained in the Julia set anymore, we have to construct curves in the Julia set which are contained in both $\partial A^{+}$and $\partial A^{-}$. Let

$$
F(t)=\int_{0}^{t} e^{s^{2}} d s
$$

for $t \geq 0$. We denote by $B^{+}$the domain bounded by $I_{U, 0}^{+}, I_{U, 1}^{+}$and $C_{U}$, and by $B^{-}$the domain bounded by $I_{U, 0}^{+}, I_{U,-1}^{+}$and $C_{U}$. The inverse of $f$ mapping $(\mathbb{C} \backslash D) \backslash I_{L}$ to $B^{+}$is denoted by $\alpha^{+}$and the inverse of $f$ mapping $(\mathbb{C} \backslash D) \backslash I_{L}$ to $B^{-}$is denoted by $\alpha^{-}$, where $I_{L}$ is the component of $I_{0} \backslash D$ in the lower halfplane. For $t \geq 0$ and $n \in \mathbb{N}$ we define

$$
h_{n}^{+}(t)=\underbrace{\alpha^{+} \circ \alpha^{-} \circ \alpha^{+} \circ \alpha^{-} \circ \cdots \circ \alpha^{\times}}_{n}\left(i F^{n}(t)\right),
$$

where $\times=+$ if $n$ is odd and $\times=-$ if $n$ is even. Similarly, we define $h_{n}^{-}(t)$ by interchanging + and - in the definition of $h_{n}^{+}(t)$. By an argument and a calculation similar to those in [5], there exist functions $h^{+}(t)$ and $h^{-}(t)$ and $t_{0}>0$ such that $h_{n}^{+}(t) \rightarrow h^{+}(t)$ and $h_{n}^{-}(t) \rightarrow h^{-}(t)$ uniformly on compact subsets of $\left\{t \mid t \geq t_{0}\right\}$. In addition, (1) $\lim _{t \rightarrow \infty}\left|h^{ \pm}(t)\right|=\infty$, 
(2) $\lim _{n \rightarrow \infty}\left|f^{n}\left(h^{ \pm}(t)\right)\right|=\infty$ for all $t \geq t_{0}$ and (3) $h^{ \pm}(t)$ is injective with respect to $t$. We denote $\left\{h^{+}(t) \mid t \geq t_{0}\right\}$ and $\left\{h^{-}(t) \mid t \geq t_{0}\right\}$ by $H^{+}$and $H^{-}$, respectively. The claim (2) shows that $H^{ \pm}$lies in the Julia set. From the definition, it is clear that $f\left(H^{ \pm}\right) \subset H^{\mp}$. We note that $h^{+}(t)=-\overline{h^{-}(t)}$ for all $t \geq t_{0}$ by symmetry. The construction implies that $H^{+}$is contained in $B_{U, 1}$ and $H^{-}$in $B_{U,-1}$.

We choose a cross cut $\Gamma$ in $A^{+}$which intersects the imaginary axis in only one point and whose complementary component containing $A^{-}$contains both singular values. We can also assume that $\Gamma$ is symmetric with respect to the imaginary axis and intersects $\partial D$ in at most two points. Let $\gamma$ be a component of $f^{-1}(\Gamma)$. Then $\gamma$ is a cross cut in $A^{-}$such that either $\gamma \cap E_{U} \neq \emptyset$ and $\gamma \cap E_{L}=\emptyset$, or $\gamma \cap E_{U}=\emptyset$ and $\gamma \cap E_{L} \neq \emptyset$. We denote the $\gamma$ intersecting $I_{U, i}$ by $\gamma_{i}$, and the complementary component of $\gamma_{i}$ which does not intersect $E_{L}$ by $G_{i}$. Since $a<0$, every unbounded subset of $I_{U, i}^{-}$intersects $G_{i}$. We see that $H^{ \pm}$is contained in $\left(G_{i}\right)^{\mathrm{c}}$ for $i \in \mathbb{Z} \backslash\{0\}$.

Let $w$ be the intersection point of $I_{U, 1}$ and $\gamma_{1}$, which is in $A^{-}$. Take a subcurve $\gamma^{\prime}$ of $\gamma_{1}$ whose finite terminal point is $w$. Let $\gamma^{\prime \prime}$ be an asymptotic path for $a^{-}$in $A^{-} \cap\{z \mid \Re z>0\}$ whose finite terminal point is $w$. We denote $\gamma^{\prime} \cup \gamma^{\prime \prime}$ by $\sigma_{1}$. In a similar fashion, we construct a cross cut $\sigma_{-1}$ in $A^{-}$. Let $\sigma_{0}$ be a cross cut in $A^{-} \cap\left(E_{U} \cup E_{L}\right)^{\mathrm{c}}$ such that one side of $\sigma_{0}$ is an asymptotic path for $a^{+}$, the other side is an asymptotic path for $a^{-}$, and $\sigma_{0} \cap\left(\sigma_{-1} \cup \sigma_{1}\right)=\emptyset$. Let $G$ be a domain bounded by $\sigma_{1}, \sigma_{0}$ and $\sigma_{-1}$ which intersects $A^{+}$. Then every component of $f^{-1}\left(A^{-}\right)$except $A^{+}$is contained in $G^{\mathrm{c}}$, and $H^{ \pm}$is contained in $G$.

Take a point $w$ in $H^{+}$. Suppose that $w$ is in neither $\partial A^{+}$nor $\partial A^{-}$. Then we can take a neighborhood $V$ of $w$ so that $V \cap A^{+}=\emptyset$ and $V \cap A^{-}=\emptyset$. Let $N$ be the smallest number satisfying $f^{N}(V) \cap A^{-} \neq \emptyset$. Since the inverse image of $A^{+}$is $A^{-}$, we see that $f^{n}(V) \cap A^{+}=\emptyset$ for all $n \leq N$. Because $f^{n}(V) \cap\left(H^{+} \cup H^{-}\right) \neq \emptyset$ for all $n$, there exists $\sigma_{i}$ for some $i \in\{0, \pm 1\}$ such that $\sigma_{i} \cap f^{N-1}(V) \neq \emptyset$. This is a contradiction because $\sigma_{i} \subset A^{-}$. Hence $w \in \partial A^{+}$ or $w \in \partial A^{-}$. If there exists $\left\{w_{n}\right\} \subset \partial A^{-} \cap H^{+}$such that $\lim _{n \rightarrow \infty} w_{n}=w$, then we see that $w \in \partial A^{-} \cap H^{+}$. Assume that there exists a subarc $\ell$ in $H^{+}$such that $\ell \subset \partial A^{+}$and $\ell \cap \partial A^{-}=\emptyset$. Let $\ell^{\prime}$ be the reflection of $\ell$ with respect to the imaginary axis. It is clear that $\ell^{\prime} \subset H^{-}$and $\ell^{\prime} \subset \partial A^{+}$by the symmetry of the Fatou set. If $\ell$ is unbounded, then $f(\ell) \cap \ell^{\prime} \neq \emptyset$ is the desired curve because $f(\ell) \subset H^{-} \cap \partial A^{-}$. We take the component of $\left(\overline{A^{+}} \cup \overline{A^{-}}\right)^{\mathrm{c}}$ whose boundary contains $\ell$, and denote it by $V$. There exists a smallest $N$ such that $f^{N}(V) \cap A^{-} \neq \emptyset$. It follows that $f^{N-1}(V)$ intersects a component of $f^{-1}\left(A^{-}\right)$other than $A^{+}$. From $\partial f^{N-1}(V) \cap\left(H^{+} \cup H^{-}\right) \neq \emptyset$ we conclude that $f^{N-1}(V) \cap A^{-} \neq \emptyset$. This is a contradiction. Hence if $w \in \partial A^{+} \cap\left(H^{+} \cup H^{-}\right)$, then $w \in \partial A^{-} \cap\left(H^{+} \cup H^{-}\right)$. Accordingly, if $w \in \partial A^{-} \cap\left(H^{+} \cup H^{-}\right)$, then 
$f(w) \in \partial A^{+} \cap\left(H^{+} \cup H^{-}\right)$and thus $f(w) \in \partial A^{-} \cap\left(H^{+} \cup H^{-}\right)$. Let $t_{1}$ be the number satisfying $h^{-}\left(t_{1}\right)=f\left(h^{+}\left(t_{0}\right)\right)$. Then it is clear that

$$
H^{+}=\left(\bigcup_{n=0}^{\infty} f^{2 n}\left(h^{+}\left[t_{0}, t_{1}\right]\right)\right) \cup\left(\bigcup_{n=0}^{\infty} f^{2 n+1}\left(h^{-}\left[t_{0}, t_{1}\right]\right)\right) .
$$

Therefore, we conclude that $\left\{h^{+}(t) \mid t \geq t_{1}\right\}$ and $\left\{h^{-}(t) \mid t \geq t_{1}\right\}$ are common boundary curves.

CASE 2: $B>0$. From the symmetry of the action of $f$, we see that the real axis is forward invariant. Both asymptotic values exist on the real axis. However, the imaginary axis is not forward invariant anymore. We recall that $b=\sqrt{B}$ and denote $\{z \mid \Re z=b\}$ by $I_{1}$. Then $f^{-1}\left(I_{1}\right)$ coincides with $K$ defined in Case 1. Since $D$ defined in Case 1 contains both asymptotic values, we see that $D \cap I_{1} \neq \emptyset$. Therefore, the notation derived from $D$ and $K$ in Case 1 is in force again.

First, we consider the case where $f$ has two attracting fixed points. It follows that $a>0$. Let $A^{+}$and $A^{-}$be the immediate basins containing $a^{+}$ and $a^{-}$, respectively. We denote by $B_{U}$ the domain bounded by $I_{U, 1}^{-}, I_{U,-1}^{-}$ and $C_{U}$. The inverse of $f$ mapping $(\mathbb{C} \backslash D) \backslash I_{L}$ to $B_{U}$ is denoted by $\beta$, where $I_{L}$ is the component of $I_{1} \backslash D$ in the lower halfplane. For $t \geq 0$ and $n \in \mathbb{N}$ we define

$$
h_{n}(t)=\beta^{n}\left(i F^{n}(t)\right) .
$$

As was stated before, there exist a function $h(t)$ and $t_{0}$ such that $h_{n}(t) \rightarrow$ $h(t)$ uniformly on compact subsets of $\left\{t \mid t \geq t_{0}\right\}$. We denote $\left\{h(t) \mid t \geq t_{0}\right\}$ by $H$. Let $\Gamma^{+}=\left[a^{+},+\infty\right)$ and $\Gamma^{-}=\left(-\infty, a^{-}\right]$. We choose the component of $f^{-1}\left(\Gamma^{+}\right)$which is an asymptotic path for $a^{+}$and intersects $B_{U, 1}$, and denote it by $\gamma_{U}^{+}$. We also choose the component of $f^{-1}\left(\Gamma^{-}\right)$which is an asymptotic path for $a^{-}$and intersects $B_{U,-1}$, and denote it by $\gamma_{U}^{-}$. The inverse of $f$ mapping the upper halfplane to the domain bounded by $\gamma_{U}^{+}, \gamma_{U}^{-}$ and the real axis is denoted by $\widetilde{\beta}$; it coincides with $\beta$ on $(\mathbb{C} \backslash D) \cap\{z \mid \Im z>0\}$. Since $\widetilde{\beta}$ is a contraction map, it has an attracting fixed point on the real axis, say $a_{0}$, which is a repelling fixed point of $f$. From the definition, we see that $\widetilde{\beta}(H) \supset H$. Hence $S^{+}:=\bigcup_{n=0}^{\infty} \widetilde{\beta}^{n}(H)$ is the curve contained in the Julia set whose finite end point is $a_{0}$. Furthermore, $f\left(S^{+}\right)=S^{+}$. The reflection of $S^{+}$with respect to the real axis is denoted by $S^{-}$. It is a curve in the Julia set. The curve $S:=S^{+} \cup S^{-}$is a cross cut one of whose complementary components contains $A^{+}$and the other contains $A^{-}$. Hence every component of $f^{-1}\left(A^{+}\right)$is contained in the complementary component of every component of $f^{-1}(S)$. The same is true for $A^{-}$. We choose the component of $f^{-1}\left(\Gamma^{+}\right)$which is an asymptotic path for $a^{+}$and intersects $B_{L, 1}$, and denote it by $\gamma_{L}^{+}$. We also choose the component of $f^{-1}\left(\Gamma^{-}\right)$which is an asymptotic path for $a^{-}$and intersects $B_{L,-1}$, and denote it by $\gamma_{L}^{-}$. 
Every component of $f^{-1}\left(A^{+}\right)$and $f^{-1}\left(A^{-}\right)$except $A^{ \pm}$is contained in the complement of the domain bounded by $\gamma_{U}^{+}, \gamma_{U}^{-}, \gamma_{L}^{+}$and $\gamma_{L}^{-}$. By the argument similar to the above, we see that every point in $S$ is in $\partial A^{+}$or $\partial A^{-}$. If there were a subarc on $S$ contained in $\partial A^{+}$and not intersecting $\partial A^{-}$, then we obtain a contradiction by an argument similar to the above. Hence, $S$ is a curve in $\partial A^{+}$and in $\partial A^{-}$.
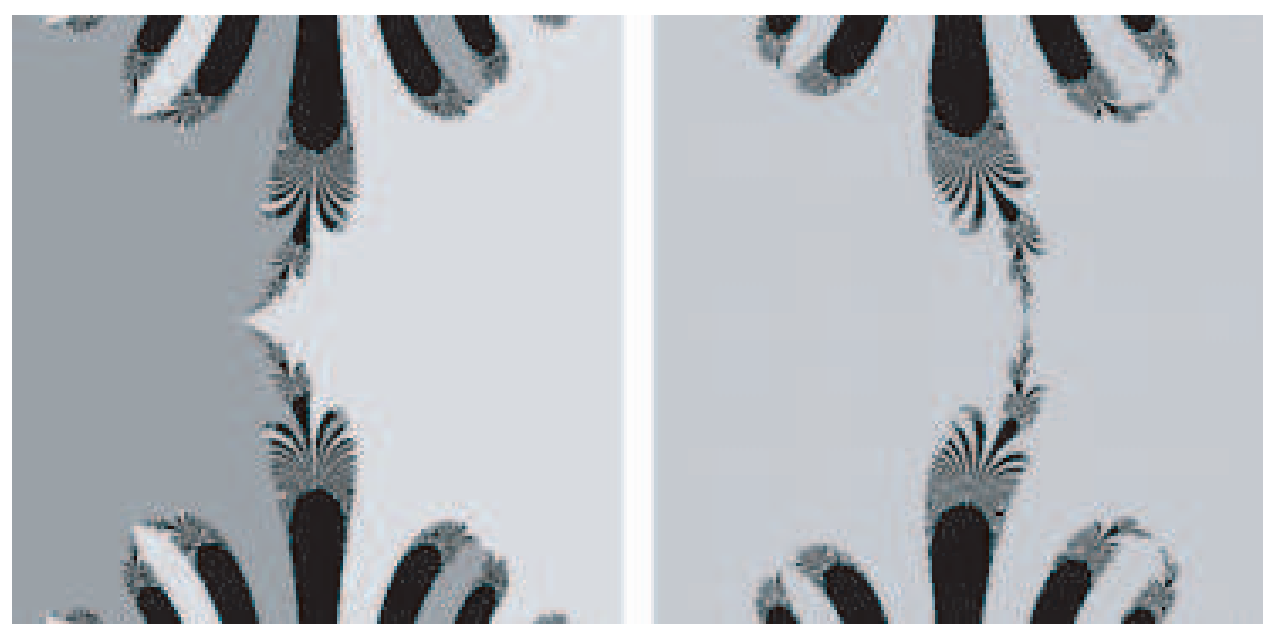

Fig. 3. The Julia sets of $f_{a, \sqrt{B}}$. Left: $f_{a, \sqrt{B}}$ with $(a, B)=(2,0.25)$ has two attracting fixed points. Right: $f_{a, \sqrt{B}}$ with $(a, B)=(-2,2.25)$ has an attracting cycle with period two.

Second, we consider the case where $f$ has an attracting cycle with period two. It follows that $a<0$. The inverse of $f$ mapping $(\mathbb{C} \backslash D) \backslash I_{U}$ to $B_{U}$ is denoted by $\beta^{+}$, where $I_{U}$ is the component of $I_{1} \backslash D$ in the upper halfplane. We denote by $B_{L}$ the domain bounded by $I_{L, 1}^{-}, I_{L-1}^{-}$and $C_{L}$. The inverse of $f$ mapping $(\mathbb{C} \backslash D) \backslash I_{L}$ to $B_{L}$ is denoted by $\beta^{-}$. By using the functions given by

$$
h_{n}^{+}(t)=\underbrace{\beta^{+} \circ \beta^{-} \circ \beta^{+} \circ \beta^{-} \circ \cdots \circ \beta^{\times}}_{n}\left(i F^{n}(t)\right),
$$

where $\times=+$ if $n$ is odd and $\times=-$ if $n$ is even, and

$$
h_{n}^{-}(t)=\underbrace{\beta^{-} \circ \beta^{+} \circ \beta^{-} \circ \beta^{+} \circ \cdots \circ \beta^{\times}}_{n}\left(i F^{n}(t)\right) \text {, }
$$

where $\times=-$ if $n$ is odd and $\times=+$ if $n$ is even, we obtain the cross cut in a similar fashion. Hence the rest of the proof is similar to the above.

REMARK 2. The curves constructed in the proof are called ray tails. Ray tails for exponential maps have been considered by many authors, for example, [2] and [11]. In [5], Kisaka considered ray tails for structurally finite transcendental entire functions. 
REMARK 3. The author believes that the result is also true for some complex error functions, and it can be proved by detailed investigation of ray tails and their landing properties. However, it may not be true when a complex error function has only one attracting cycle with period two whose basin contains both asymptotic values. For example, the complex error function with $a=-0.7+0.7 i$ and $b=0.31-0.96 i$ has an attracting cycle with period two whose basin contains both asymptotic values and whose immediate basin contains only one asymptotic value. A numerical experiment seems to show that there exists no common curve in the boundaries of Fatou components.

\section{References}

[1] I. N. Baker, Wandering domains in the iteration of entire functions, Proc. London Math. Soc. (3) 49 (1984), 563-576.

[2] R. L. Devaney and M. Krych, Dynamics of $\exp (z)$, Ergodic Theory Dynam. Systems 4 (1984), 35-52.

[3] A. E. Eremenko and M. Yu. Lyubich, Dynamical properties of some classes of entire functions, Ann. Inst. Fourier (Grenoble) 42 (1992), 989-1020.

[4] L. R. Goldberg and L. Keen, A finiteness theorem for a dynamical class of entire functions, Ergodic Theory Dynam. Systems 6 (1986), 183-192.

[5] M. Kisaka, Dynamics of structurally finite transcendental entire functions, in: Studies on Complex Dynamics and Related Topics, Sûrikaisekikenkyûsho Kôkyûroku 1220 (2001), 17-25.

[6] S. Morosawa, On the residual Julia sets of rational functions, Ergodic Theory Dynam. Systems 17 (1997), 205-210.

[7] - , The moduli space of complex error functions with real coefficients, in preparation.

[8] S. Morosawa, Y. Nishimura, M. Taniguchi, and T. Ueda, Holomorphic Dynamics, Cambridge Stud. Adv. Math. 66, Cambridge Univ. Press, 2000.

[9] S. Morosawa and M. Taniguchi, Dynamics of structurally finite entire functions with two singular values, in preparation.

[10] P. J. Rippon and G. M. Stallard, Iteration of a class of hyperbolic meromorphic functions, Proc. Amer. Math. Soc. 127 (1999), 3251-3258.

[11] D. Schleicher and J. Zimmer, Escaping points of exponential maps, J. London Math. Soc. (2) 67 (2003), 380-400.

[12] M. Taniguchi, Explicit representation of structurally finite entire functions, Proc. Japan Acad. Ser. A 77 (2001), 68-70.

Department of Mathematics and Information Science

Faculty of Science

Kochi University

Kochi, 780-8520, Japan

E-mail: morosawa@math.kochi-u.ac.jp

Received 16 April 2004;

in revised form 23 July 2004 